\title{
Prototype of Emotional Metaphor in Chinese
}

\author{
Hong $\mathrm{Xu}^{12 *}$ \\ ${ }^{1}$ College of Foreign Languages and Cultures, Sichuan University, Chengdou, Sichuan, China; \\ ${ }^{2}$ School of Foreign Languages, Hubei Minzu University, Enshi, Hubei, China \\ ${ }^{*}$ Corresponding author. Email: 258106276@qq.com
}

\begin{abstract}
Emotional metaphor is a way of expressing and recognizing emotion, which is formed by the similarity of emotion category and other domain category. In the emotional metaphor proposition "A is B", B is a set of multiple possible values. Some categories related to individual evaluation, physiological response and behavioural action are likely to be the prototype of emotional metaphor. Types of emotional metaphor prototype in Chinese include the physiological response, sensory experience, natural phenomena, and spatial relations. Metaphor prototypes of emotional language expression provide references for embodied cognition of metaphor.
\end{abstract}

Keywords: Prototype, Emotional Metaphor, Embodied Cognition, Chinese

\section{INTRODUCTION}

Emotion is human's subjective reflection to the objective world. It contains a great deal of emotional language expression in Chinese. Psychologist Ortony and other scholars have proposed two kinds of emotionrelated words, one is "directly referring to emotion", the other is "implicitly referring to emotion", which indirectly describes emotion, namely emotional metaphor [1]. Due to the abstraction, ambiguity and complexity of emotion, people often use emotional metaphor to some extent. Since Lakoff and Johnson proposed conceptual metaphor [2], metaphor is not only considered as a linguistic rhetoric, but also a cognitive thinking mode of human beings. In everyday language, emotional metaphor is to express abstract emotion through specific source domain and form a cognitive relation between different concepts. Many studies on emotional concepts in cognitive linguistics and social anthropology have explored whether the conceptualization of human emotion is universal, while some considers it universal and others regard it sociocultural. Most studies have shown that emotional concepts are based on both universal human experience and specific socio-cultural structures. Emotional metaphor is not generated arbitrarily, but from human embodied experience, that is the result of gradual development of cognition through the repeated experience of the real world [3]. Based on this, this paper discusses the emotional metaphor prototype in Chinese in order to provide some references for the embodied cognition of emotional metaphor.

\section{EMOTION}

Emotion, as the basic state of human mind, is people's spiritual response to some important external or internal events, causing people's psychological feeling of pleasure or pain. Descartes believed that love, hate, joy, sadness, praise and expectation were the basic emotions from which other emotions were derived. Spinoza proposed that the basic emotions are happiness, sadness, and desire. The Doctrine of the Mean classifies emotions into four categories: happiness, anger, sorrow and joy. Lu Shi Chun Qiu mentions five categories: happiness, anger, sadness, fear and sorrow. Xunzi gives six categories: good, evil, happiness, anger, sorrow and joy. In general, the basic emotion category is the same.

In the field of contemporary philosophy of mind, three types of emotional theories have been formed, namely, the theory of feeling, the theory of motivation and the theory of evaluation. James was the first to explore emotions and feelings in depth, arguing that emotions are how we feel about changes in the body as they happen. Dewey rejected the theory of feeling and proposed the theory of motivation, arguing that emotion is a purposeful behavior pattern that reflects feelings. The evaluation theory, mainly represented by Arnold, believes that emotion is essentially the intuitive tendency to evaluate something as good or bad. At present, the three classical theories of emotion are gradually converging. The basic 
emotion, "anger", for example, can be defined as the core emotional state, representing an individual's negative evaluation of the outside world, leading to the psychological feeling of extreme dissatisfaction and behavioural actions, such as increased blood pressure, rapid heartbeat, red face and shouting. Thus, the concept of emotion can be understood as the psychological feelings including a series of physical response and behavior actions resulting from the individual's evaluation of objective events.

The meaning of most linguistic signs is not based on a single one, but on a group of interrelated fields. A linguistic sign obtains its meaning by highlighting a certain part or a combination of several parts in the related fields [4]. In daily language, emotional expression also has the semantic feature of the concept of emotion, including the individual's evaluation of objective world, physiological response and behavioural action. In an ideal situation, individual evaluation, physiological response and behavioural action are the archetypal features of emotional expression, which constitute the semantic field of emotional expression. It is generally believed that the core emotional words are happiness, anger, sorrow, joy, sadness, fear and surprise. Complex emotional words are far from the core emotional words, belonging to the non-core emotional words, such as, miss, calm, satisfaction, confusion, confusion. The marginal emotional words are the most inexplicable and complex. All the representations with the core emotional words are close to the prototype.

\section{EMOTIONAL METAPHOR}

Shu Dingfang proposed that similarity is the degree of similarity between two things, including physical similarity and psychological similarity, while physical similarity refers to a kind of similarity in shape, appearance or function, and psychological similarity refers to the similarity that the speaker or hearer thinks it exists in some aspects because of culture, legend or other psychological factors [5]. In the metaphorical proposition "A is B", A and B are different categories. When A and $\mathrm{B}$ are similar in some aspects or $\mathrm{A}$ and $\mathrm{B}$ are not similar in reality but similar by imagination, " $\mathrm{A}$ is $\mathrm{B}$ " is a metaphorical expression. Emotional metaphor is a type of metaphorical expression, by which people imagine the affective category and other categories to generate similar semantics according to people's cognition. Any target domain can be represented by a series of source domains. In the emotional metaphor proposition " $\mathrm{A}$ is B", A is the target domain, B is the source domain, and B is a set of all possible values, such as B1, B2, B3, and so on. Thus, it is also possible to form many metaphorical expressions: "A is B1", "A is B2", "A is B3". For example, in the proposition "A is B", $\mathrm{A}$ is "Happiness", and B1 can be "Breeze", when the speaker gives the expression "Happiness is breeze", people also gain a new understanding of "happiness", making people comfortable and comfortable. A and B have a similar concept, the metaphorical proposition "Happiness is breeze" is born. Also, if in the proposition "A is B", A is happiness and B2 can be honey. People have experienced the emotional state of happiness, at the same time, they have eaten honey and experienced the pleasure of it, then the metaphorical expression "happiness is honey" is recognized and accepted. In the proposition " $\mathrm{A}$ is $\mathrm{B}$ ", it can also be assumed that $\mathrm{A}$ is happiness and $\mathrm{B} 3$ can be "up". Since man is an upright walking animal, he stood straight when happy, so the "happiness is up" metaphor is accepted but sometimes not realized as a metaphorical expression. When metaphorical expression is accepted by most people, it gradually becomes conventional metaphor, that is, the language use in daily life

In general, emotional metaphors are not arbitrary but largely determined by similar features. According to the feature of similarity, people can grasp the uncertain and imaginary semantic category of source domain based on the target domain. In the proposition "A is B", if the semantic category of $B$ is related to emotion, such as the individual evaluation, physiological response and behavioural action, it is likely to be highlighted, and the proposition " $\mathrm{A}$ is $\mathrm{B}$ " is also likely to become emotional metaphor, while those without these features or with fewer features are unlikely to become emotional metaphor. For the possibilities of B in reality, the emotional metaphor can be shown in figure 1.

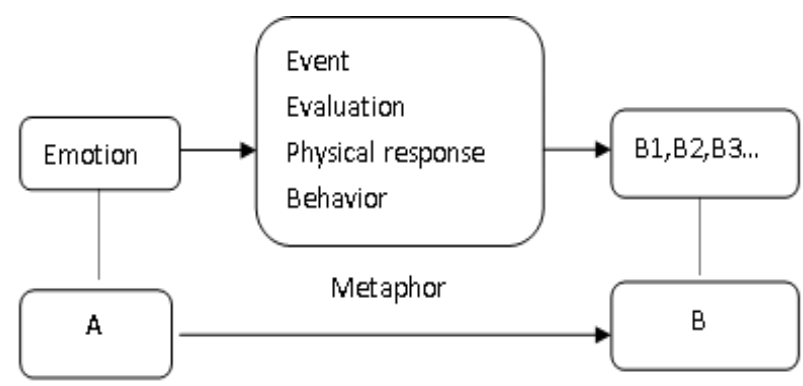

Figure 1 Emotional Metaphor

Here, it is necessary to notice that emotional metaphor is not only one, of course, and may have multiple prototypes. Kövecses proposed that the source domain of emotional metaphor mainly includes a series of archetypal scenarios, such as "emotion is a container" [6]. For example, the prototypes of "anger" metaphor can be "anger is fire", "anger is gas", "anger is a physiological effect". People can make good or bad evaluations of these emotions, which may also lead to corresponding physiological reactions and behavioural actions. 


\section{TYPES OF EMOTIONAL METAPHOR PROTOTYPE IN CHINESE}

\subsection{Physiological Response}

Due to the common physiological structure, when people are in a certain emotional state, they often have special physiological reactions, which affect internal organs such as heart, lung, spleen, liver, gallbladder, intestine and abdomen. In traditional Chinese medicine, the five elements of metal, wood, water, fire and earth correspond to five viscera that control five emotions respectively: the lung predominates in sadness, the liver predominates in anger, the kidney predominates in fear, the heart predominates in happiness, and the spleen predominates in anxiety. Many conventional emotional expressions in Chinese use various parts of the body. "Xin" means "Heart", which is the frequently used body in expressing emotion, such as, "kai xin", "xin sui", "xin hua nu fang", "xin tou huo qi". Emotional metaphors transform abstract emotional concepts into concrete physiological phenomena through the experience of specific body organs.

\subsection{Sensory Experience}

Sensory experience is people's sensory judgment of objective things, mainly including vision, hearing, smell, taste and touch. Due to the mutual influence between different sensory organs, people often use synesthesia to transfer the words of one sense to express another feeling when describing objective world, while the words of various sensory categories are also used to express people's special emotion. In terms of "taste", there are lots of similar emotional language expressions in Chinese, such as, "tian mi mi", "xin suan", "tong ku". Also, in various emotional states, the human body temperature also changes naturally, and the concept of temperature is often used to metaphorically understand the emotional field, such as, "nuan xin", "leng ku". Sensory experience can be used as a metaphor for people's complex emotions.

\subsection{Natural Phenomena}

Human beings' subjective reactions to objective world provide an experiential basis for the formation of emotional metaphors. Weather is one of the most basic phenomena in the objective world. "Lei" is the thunder producing electrical discharge, which is considered to be a trigger danger, and anger can also cause damage to those around. For example, "da fa lei ting" and "bao tiao ru lei" are the vivid descriptions of the emotion in Chinese, causing a frightening effect. In Chinese, "huo" is the fire that can be evaluated as bad and dangerous things, used to express inner dissatisfaction, such as "fa huo", "nao huo", "nu huo" and other emotional expressions. In traditional Chinese culture, "qi" is the air believed to be the energy of life, used to express "anger", such as "sheng qi", "qi si le", "qi zha le". Also, "guang" is the light or brightness used to describe the emotion of "joy", such as "rong guang huan fa", "man mian hong guang". The change of external weather indicates the change of individual emotion.

\subsection{Spatial Relations}

Spatial relation is the expression form of the existence of objective world. People understand and experience the world through the spatial relationship between them and the outside world, so spatial emotional metaphor has become one of the important fields in which people express their emotions. In spatial relationships, Up and down usually have positive or negative evaluations of emotional and physical states. In terms of "up", some emotional expressions can be found in Chinese, such as "ti qi xing zhi”, "qing xu gao zhang". In terms of "down", there are also some emotional expressions, such as "qing xu di luo", "xiao chen". Emotional metaphors have something to do with spatial relations.

\section{THE SEMANTIC FEATURES OF THE PROTOTYPE OF EMOTIONAL METAPHOR}

The concept of emotion covers the process of psychological experience in which an individual evaluates an objective event to produce a series of physical feelings and behavioural actions. In daily language, the expression of emotion is also in the semantic field of the concept of emotion, which includes the following semantic features: in the objective event domain, the emotion is the psychological experience of the external objective event; and in the individual evaluation domain, emotion is a psychological experience that an individual evaluates as good or bad; in the physiological response domain, it is a psychological experience of a complex physiological response; and in the behavioural action domain, it is a psychological experience in which emotions cause actions. Those expressions containing these semantic features do not necessarily express emotion. At the same time, emotional language expression do not necessarily contain all semantic features, but they must contain one or more of them, and connect with each other through family resemblance.

When the metaphorical proposition "A is B" is said, it implies that " $\mathrm{A}$ is $\mathrm{B}$ " is true in imaginary world, then $\mathrm{A}$ and $B$ must have similar semantic feature in the imaginary world. According to the theory of cognitive category, their common semantic feature must be not random but in a special semantic field, including the evaluation of objective events, physiological responses, behavioural actions and so on. All these possible semantics also constitute the possible semantic field of metaphor 
understanding. In the same emotional state, there are different metaphorical archetypes of emotion. For example, the prototype of emotional metaphor, "anger is fire", contains the semantic features of anger, such as outburst, danger, process and intensity.

The meaning of a word symbol is a fragmentation and static abstraction of a concept, while "fragmentation" is an abstract representation of a meaning component or part of a concept, "static" is a representation when a word solidifies an element or part of an intercepted concept by social convention [7]. The proposition "A is B" reflects the semantic similarity between different categories. If " $A$ " is emotional anger, and " $\mathrm{B}$ " is fire, then the emotional metaphor "anger is fire" shows that "anger" and "fire" have a similar meaning. That is, when people understand the emotional metaphor of "anger is fire", people will think of the intensity, danger, and destructiveness of the fire instead of thinking fire as something to cook and eat, or as a tool to warm people. The similar semantic can be the "fragmentation" of concepts, that is, "anger is fire" implies the comparison of the intensity and harmfulness of anger to the intensity and destructiveness of fire, describing to what extent the harm is and where the harm lies. At the same time, the similar semantic can also be the "static" of the concept. For example, "huo mao san zhang" has gradually been accepted by people as a metaphor for the emotional state of "anger" instead of "qi mao san zhang", which is also used to say "qi zha le", instead of saying "huo bao le". As long as " $\mathrm{A}$ is $\mathrm{B}$ " is said, it implies that $\mathrm{A}$ and $\mathrm{B}$ have similar semantic, and the semantic is the "fragmentation" of the concept, the abstract representation of the concept, or the "static", which is fixed by social conventions.

\section{CONCLUSION}

The emotional metaphor prototype in Chinese provides a justification for the embodied experience of metaphor. Logically, people have some emotional experience in the interaction with the external world, and this emotional experience is similar to the way people understand the world. It is the similarity that generates emotional metaphor. In the proposition of "A is B" of emotional metaphor, $\mathrm{B}$ is a set of various possible values. If its semantic features are similar to those of individual evaluation, physiological response and behavioural action, it is more prominent and most likely to become the prototype of emotional metaphor. Types of emotional metaphor prototype in Chinese include the physiological response, sensory experience, natural phenomena, and spatial relations. Emotional metaphor is the result of the combination of people's own emotional experience and understanding of the objective world.

\section{REFERENCES}

[1] A. Ortony, G. Clore, M. Foss, The referential structure of the affective lexicon, Cognitive
Science, (11) (1987) 341-364. DOI:

https://doi.org/10.1207/s15516709cog1103_4

[2] G. Lakoff, M. Johnson, Metaphors we live by, University of Chicago Press, 20003. DOI: https://doi.org/10.7208/chicago/9780226470993.00 1.0001

[3] Sun Yi, The embodied origin of core emotional metaphor, Journal of Shanxi Normal University, (1) (2013) 105-111.

[4] Lan Chun, Cognitive linguistics and metaphor, Foreign Language Teaching and Research Press, 2005.

[5] Su Dingfang, The study of metaphor, Shanghai Foreign Language Education Press, 2000.

[6] Z. Kövecses, Emotion concept, Springer-Verlag, 1990. DOI: https://doi.org/10.1007/978-1-4612 3312-1

[7] Liu Liming, What is the meaning given by "ma"?-A study on the minimum semantic of words, Journal of Foreign Languages, (1) (2019): 7-10. DOI : 10.16263/j.cnki.23-1071/h.2019.01.002 